\title{
Assessment of Inter-Characters Associations in the Germplasm of Pearl Millet [Pennisetum glaucum (L.) R. Br.] Over Five Years in Hot Arid Climate of Rajasthan, India
}

\author{
Om Vir Singh", R. Gowthami, Kartar Singh and Neelam Shekhawat \\ ICAR-National Bureau of Plant Genetic Resources Regional Station, Jodhpur-342003, India \\ *Corresponding author
}

\begin{tabular}{|l|}
\hline K e y w o r d s \\
Pearl millet, \\
$\begin{array}{l}\text { Genetic variability, } \\
\text { Heritability, Inter } \\
\text { characters } \\
\text { associations }\end{array}$ \\
\hline Article Info \\
\hline $\begin{array}{l}\text { Accepted: } \\
\text { 26 December } 2017 \\
\text { Available Online: } \\
\text { 10 January 2018 }\end{array}$ \\
\hline
\end{tabular}

\section{A B S T R A C T}

Forty accessions of pearl millet were evaluated for five consecutive years (environments) to assess the magnitude of presence of genetic variability, heritability in broad sense, genetic advance, inter characters correlation and path coefficients for yield and yield contributing characters. All the accessions showed considerable amount of variation in their mean performance with respect to studied characters. The phenotypic co-efficient of variation (PCV) was greater than genotypic co-efficient of variation (GCV) for all the studied characters this shows the influence of environmental factors on the expression of characters. High GCV and PCV estimates were observed for plant height, total number of tillers per plant, number of productive tillers per plant, number of leaves per plant, spike girth, ear exertion distance, test weight, and yield per plant in all the environments. High heritability coupled with high genetic advance was observed for plant height, total number of tiller per plant, number of productive tillers per plant, number of leaves per plant, spike length, spike girth, ear exertion distance, test weight, yield per plant, stover yield per plant and harvest index in all the environments. Seed yield per plant was positively and significantly correlated with number of productive tillers per plant, number of leaves per plant, spike length, spike girth, test weight, stover yield and harvest index in all the environments and in pooled environment. Genotypic path coefficient analysis revealed highest positive direct effect registered by spike girth, spike length, number of productive tillers per plant, plant height and test weight in all the environments. Hence, these characters have to be given importance during the selection programme to improve the seed yield potential of the crop.

\section{Introduction}

Pearl millet [Pennisetum glaucum (L.) R. Br.] is an outstanding dual purpose cereal crop with enormous variability for agronomically important characters adapted to diverse agroclimatic conditions. Due to its potential to with stand drought and adverse agro climatic conditions, it is mainly grown under marginal lands with low rainfall during Kharif season (Vidyadhar et al., 2007) and (Bhoite et al., 2008). Estimation of genetic parameters is an essential component of future targeted trait based crop improvement. The measurement and evaluation of variability are essential in drawing essential steps and meaningful 
conclusion from a given set of phenotypic observations (Mehdi and Khan., 1994; Marwede et al., 2004). Hence, to have a thorough comprehensive idea it is necessary to have an analytical assessment of yield components. Since heritability is also influenced by environment thus, information on heritability alone may not help in pin pointing characters enforcing selection. Nevertheless the heritability estimates in conjunction with the predicted genetic advance will be more reliable (Johnson et al., 1955). Heritability gives the information on the magnitude of inheritance of quantitative characters while genetic advance will be helpful in formulating suitable breeding procedures.

Grain yield in pearl millet is a complex character controlled by many factors and is the final product of actions and interactions of various yield contributing characters hence, understanding association between yield and its components is of paramount importance. In order to develop promising accessions with high yield it is essential to know the associations among different traits especially with seed yield. The correlation and path analysis are usually taken up to measure the relative magnitude and direction of each independent variable on a dependent variable like seed yield. Therefore, the present research work was attempted to understand presence of nature and magnitude of genetic variability, heritability and genetic advance under selection of different yield and yield contributing characters and also pattern of character associations of yield and yield contributing traits in germplasm of pearl millet.

\section{Materials and Methods}

Forty pearl millet [Pennisetum glaucum (L.) R. Br.] accessions, collected from diverse geographical regions of country and conserved at Regional Seed Gene Bank at ICARNBPGR, Regional Station, Jodhpur, along with checks were evaluated at the research farm of NBPGR Regional Station, Jodhpur, which is situated at about $28^{\circ} 35^{\prime} \mathrm{N}$, longitude of $70^{\circ} 18^{\prime} \mathrm{E}$ and an altitude of $226 \mathrm{~m}$ above mean sea level. These accessions viz., IC 285152, IC 285200, IC 323995, IC 324035, IC 325176, IC 325739, IC 325804, IC 329029, IC 329070, IC 329909,IC 333121,IC 333179, IC 333240, IC 369836, IC 370487, IC 370507, IC 373424, IC 373504IC 373558, IC 420330, IC 420367, IC 426704, IC 426811, IC 426892, IC 426907, IC 449439, IC 449474, IC 537957, IC 537996, IC 538001, IC 541018, IC 541900, NIC 17769, NIC 17795, NIC 17819, JBV-2, CZP-9802, Pusa-383, Raj-171) represented diverse geographic regions of India. The trials were conducted in a Randomized Complete Block Design (RBD) with three replications for five consecutive years (environments) viz., Kharif 2012 (E1), Kharif 2013 (E2), Kahrif 2014 (E3), Kharif 2015 (E4) and Kharif 2016 (E5). The line to line and plant to plant distances were kept 45 and $15 \mathrm{~cm}$, respectively. The recommended agronomic packages of practices were followed to raise good crop during the experimental period. Fourteen morphological traits (days to fifty percent flowering, days to maturity, days for reproductive period, plant height $(\mathrm{cm})$, total number of tillers per plant, number of productive tillers per plant, number of leaves per plant, ear exertion distance $(\mathrm{cm})$, spike length $(\mathrm{cm})$, spike girth $(\mathrm{cm})$, test weight $(\mathrm{g})$, stover yield per plant(g), seed yield per plant (g) and harvest index (\%) were recorded on five randomly taken plants of each plot as per the standard descriptors, developed for pearl millet. The mean of all the traits of plants in each replication was subjected to analysis of variance as per the method suggested by Panse and Sukhathme (1967). The estimate of genotypic variance and phenotypic variance were worked out according to the method suggested by Johnson et al., (1955) using 
mean square values from the ANOVA table. Phenotypic and genotypic coefficients of variance were calculated based on the method given by Burton (1952). Heritability in broad sense was estimated as per the method described by Lush (1940) and traits were classified as having high, moderate and low heritability as per the method of Robinson et al., (1949). Genetic advance was estimated according to the method suggested by Johnson et al., (1955) and expressed as percentage of mean. Traits were classified as having high, moderate or low genetic advance as per the method suggested by Johnson et al., (1955), while correlation coefficients and path coefficient analysis were calculated using the formulae suggested by Falconer (1964) and Dewey and $\mathrm{Lu}$ (1959) respectively.

\section{Results and Discussion}

Mean performance of the accessions in different locations

The analysis of variance revealed the significant difference among the accessions for some of the major traits. Among the five environments (Table 1), E3 (51.58 days) had taken minimum days to $50 \%$ flowering followed by E1 (51.69 days), Pooled (52.24 days), E4 (52.44 days), E5 (52.59 days) and E2 (52.91 days). E1 (82.35 days) had taken minimum days to maturity followed by E3 (82.63 days), E5 (82.86 days), pooled (83.08 days), E2 (83.43 days) and E4 (84.15 days) while reproductive period was minimum in E4 (30.12 days) followed by E5 (30.75 days), pooled (30.78 days), E2 (30.88), E3 (30.90 days) and E1 (31.23 days). E4 $(192.33 \mathrm{~cm})$ was more favourable for higher plant height followed by E2 $(187.70 \mathrm{~cm})$, pooled $(183.03$ $\mathrm{cm})$, E5 $(181.01 \mathrm{~cm})$, E3 $(178.47 \mathrm{~cm})$ and E1 $(175.67 \mathrm{~cm})$. Considering the performance over four environments, E3 (2.84) was more favourable for better expression of the total number of tillers per plant followed by E4,
(2.81), E1 (2.79), pooled (2.79), E2 (2.77) and E5 (2.71) whereas number of productive tillers was maximum in E5 (2.45) followed by E3 (2.45), E2 (2.40), pooled (2.40), E1 (2.36) and E4 (2.35). Number of leaves per plant was highest in E5 (8.50) followed by E3 (8.46), pooled (8.43), E2 (8.42), E1 (8.41) and E4 (8.38), Spike length in E3 $(27.54 \mathrm{~cm})$ followed by E5 $(27.23 \mathrm{~cm})$, pooled $(26.83 \mathrm{~cm}), \mathrm{E} 1$ $(26.75 \mathrm{~cm}), \mathrm{E} 4(26.57 \mathrm{~cm})$ and E2 $(26.06 \mathrm{~cm})$, Spike girth in E4 $(1.85 \mathrm{~cm})$ followed by E2 $(1.85 \mathrm{~cm})$, pooled $(1.84 \mathrm{~cm})$, E5 $(1.84 \mathrm{~cm})$, E3 $(1.82 \mathrm{~cm})$ and E1 $(1.82 \mathrm{~cm})$, Ear exertion distance in E2 $(6.18 \mathrm{~cm})$ followed by E5 (6.15 $\mathrm{cm})$, E1 $(6.13 \mathrm{~cm})$, pooled $(6.09 \mathrm{~cm})$, E3 $(6.02$ $\mathrm{cm})$ and E4 $(5.98 \mathrm{~cm})$, test weight in E1 $(8.16$ g) followed by E5 $(8.09 \mathrm{~g})$, E4 $(8.09 \mathrm{~g})$, pooled (8.08), E3 (8.07 g) and E2 (8.01 g). Yield per plant was maximum in E4 (114.34 g)followed by E3 (113.72 g), E2 (110.77 g), pooled (109.98 g), E5 (109.32 g) and E1 (101.77 g), stover yield per plant in E1 $(490.09 \mathrm{~g})$ followed by E4 (473.27 g), pooled (18.39 g), E2 (468.70 g), E5 (463.68 g) and E3 $(452.23 \mathrm{~g})$, harvest index in E3 $(19.30 \%)$ followed by E4 (19.14\%), E5 (18.51\%), E2 $(18.45 \%)$, pooled $(18.39 \%)$ and E1 (16.55 $\%)$.

\section{Variability}

The estimates of genotypic and phenotypic coefficient of variation are necessary to understand the role of environmental influence on different traits. The differences between the GCV and PCV indicate the level of environmental variations that contributes a major part in the expression of traits (Majumdar et al., 1974). Accessions exhibited considerable amount of variability for all the fourteen studied traits over five years. The estimates of genotypic coefficients of variation were lesser than the estimates of phenotypic coefficients of variation for all the traits in all the environments indicating the environmental influence over the studied 
traits. High GCV and PCV estimates were observed for plant height, total number of tillers per plant, number of productive tillers per plant, number of leaves per plant, spike girth, ear exertion distance, test weight, and yield per plant in all the environments (Table 1 and Fig. 1). This indicated that there is greater diversity for these characters in pearl millet. Hence direct selection based on these traits would be effective. The high PCV and GCV were earlier reported in pearl millet by Kumar et al., (2014) for productive tillers per plant, grain yield per plant and panicle length traits, similarly Bhuri singh et al., (2014) reported high PCV and GCV for grain yield per plant and 1000 seed weight. These results are in conformity with the report of Vetriventhan and Nirmalakumari (2007), Dapke et al., (2014), Singh et al., (2014) and Harinarayan et al., (2015) in pearl millet. High GCV and PCV for total number of leaves were also reported by Suthamathi and Stephen Dorairaj (1995), Vidyadhar et al., (2007) and Bhoite et al., (2008).

PCV and GCV values were estimated medium for days to fifty per cent flowering, spike length and harvest index in E1, for reproductive period in E4 and for stover yield per plant in E2. Low PCV and GCV were reported for days to maturity in E2, for days to fifty per cent flowering and stover yield per plant in E4. While high PCV and medium GCV was observed in E2, E3, and E5 for reproductive period and in E5 for stover yield per plant. Medium PCV and low GCV was observed in E1, E3 and E5 for days to fifty per cent flowering and for stover yield per plant in E1 and E3. The low GCV estimates were observed for days to maturity is in confirmation with the earlier findings of Deb Choudhary and Das (1998), Saraswathi et al., (1995), Kumari and Nagarajan (2008), Lakshmana et al.,(2009), Lakshmana et al., (2010) and Chaudhary et al., (2012) in pearl millet.

\section{Heritability and genetic advance}

High heritability coupled with high genetic advance was observed for plant height, total number of tiller per plant, number of productive tillers per plant, number of leaves per plant, spike length, spike girth, ear exertion distance, test weight, yield per plant, stover yield per plant and harvest index in all the environments (Table 1). For reproductive period medium heritability and medium genetic advance was observed in E4, for plant height medium heritability and high genetic advance was observed in E5 and for the trait harvest index high heritability coupled with medium genetic advance was recorded in E3, E4 and E5, while for the trait stover yield per plant high heritability coupled with medium genetic advance was recorded in E2, E3, E4 and medium heritability and medium genetic advance was observed in E1 for these traits. High heritability coupled with high genetic advance values were reported in pearl millet by Lakshmana et al., (2009) for plant height, productive tillers per plant, ear head length and grain yield per plant. Singh et al., (2013) reported high heritability along with high genetic advance for number of tillers per plant and fodder yield per plant in sorghum. Salih et al., (2014) reported high heritability along with high genetic advance for 1000 seed weight. Govindaraj et al., (2011) reported high heritability along with high genetic advance for grain yield per plant and panicle length. Sharma et al., (2003) reported heritability along with high genetic advance for number of leaves per plant.

Characters having high heritability and high genetic advance generally indicates that heritability is more due to the additive gene effects. Yadav et al., (2001) and Singh et al., (2009) reported that high estimate of heritability along with high magnitude of genetic advance is useful for genetic improvement of any trait through selection. 
Table.1 Variability parameters of different traits in different environments (E1, E2, E3, E4, E5, and pooled environments)

\begin{tabular}{|c|c|c|c|c|c|c|c|c|}
\hline Traits & $\begin{array}{l}\text { Enviro } \\
\text { nment }\end{array}$ & Mean & Min. & Max. & $\begin{array}{l}\text { PCV } \\
(\%)\end{array}$ & $\begin{array}{l}\text { GCV } \\
(\%)\end{array}$ & $\begin{array}{l}\mathbf{h}^{2} \\
(\%)\end{array}$ & $\begin{array}{c}\text { Genetic advance } \\
\% \text { of mean }\end{array}$ \\
\hline \multirow{6}{*}{$\begin{array}{l}\text { Days to } 50 \% \\
\text { flowering } \\
\text { (DF) }\end{array}$} & E1 & 51.69 & 44.33 & 58.00 & 11.51 & 9.65 & 70.30 & 16.67 \\
\hline & E2 & 52.91 & 44.67 & 58.00 & 10.24 & 9.45 & 85.07 & 17.95 \\
\hline & E3 & 51.58 & 45.00 & 57.33 & 11.45 & 9.87 & 74.32 & 17.53 \\
\hline & E4 & 52.44 & 44.00 & 58.67 & 9.90 & 7.74 & 61.13 & 12.47 \\
\hline & E5 & 52.59 & 42.00 & 58.67 & 10.38 & 9.82 & 89.50 & 19.14 \\
\hline & Pooled & 52.24 & 44.33 & 57.73 & 10.51 & 8.96 & 72.64 & 15.73 \\
\hline \multirow{6}{*}{$\begin{array}{l}\text { Days to Maturity } \\
\text { (DM) }\end{array}$} & E1 & 82.35 & 75.33 & 89.00 & 7.58 & 6.28 & 68.56 & 10.71 \\
\hline & E2 & 83.43 & 75.33 & 89.67 & 7.71 & 7.21 & 87.43 & 13.89 \\
\hline & E3 & 82.63 & 73.00 & 92.00 & 7.75 & 6.57 & 71.85 & 11.47 \\
\hline & E4 & 84.15 & 75.33 & 92.00 & 7.45 & 7.13 & 91.63 & 14.05 \\
\hline & E5 & 82.86 & 73.67 & 92.00 & 7.79 & 6.62 & 72.19 & 11.58 \\
\hline & Pooled & 83.08 & 75.27 & 90.60 & 7.83 & 7.66 & 95.91 & 15.46 \\
\hline \multirow{6}{*}{$\begin{array}{l}\text { Reproductive } \\
\text { period (Days) }\end{array}$} & E1 & 31.23 & 28.33 & 34.33 & 17.48 & 14.32 & 67.10 & 24.16 \\
\hline & E2 & 30.88 & 28.00 & 34.00 & 21.52 & 18.50 & 73.90 & 32.76 \\
\hline & E3 & 30.90 & 26.33 & 35.00 & 21.15 & 18.99 & 80.64 & 35.13 \\
\hline & E4 & 30.12 & 26.00 & 37.33 & 17.26 & 12.86 & 55.48 & 19.73 \\
\hline & E5 & 30.75 & 27.67 & 35.67 & 21.28 & 19.14 & 80.91 & 35.47 \\
\hline & Pooled & 30.78 & 28.00 & 33.87 & 21.33 & 19.21 & 81.16 & 35.65 \\
\hline \multirow[t]{6}{*}{ Plant height $(\mathrm{Cm})$} & E1 & 175.67 & 67.32 & 259.55 & 73.99 & 58.09 & 61.64 & 93.95 \\
\hline & $\mathbf{E 2}$ & 187.70 & 72.68 & 259.96 & 60.62 & 50.58 & 69.62 & 86.94 \\
\hline & E3 & 178.47 & 84.27 & 249.92 & 69.94 & 56.84 & 66.05 & 95.16 \\
\hline & E4 & 192.33 & 73.67 & 264.32 & 63.02 & 49.92 & 62.75 & 81.46 \\
\hline & E5 & 181.01 & 84.86 & 266.40 & 70.26 & 52.16 & 55.11 & 79.76 \\
\hline & Pooled & 183.03 & 76.56 & 257.68 & 70.75 & 54.65 & 59.67 & 86.97 \\
\hline \multirow{6}{*}{$\begin{array}{l}\text { Total number of } \\
\text { tillers per plant } \\
\text { (TP) }\end{array}$} & E1 & 2.79 & 1.87 & 5.20 & 33.59 & 30.00 & 79.77 & 55.19 \\
\hline & E2 & 2.77 & 1.47 & 5.40 & 41.25 & 35.25 & 73.02 & 62.05 \\
\hline & E3 & 2.84 & 1.80 & 4.27 & 32.25 & 28.37 & 77.39 & 51.41 \\
\hline & E4 & 2.81 & 1.47 & 4.73 & 36.47 & 34.79 & 91.00 & 68.37 \\
\hline & E5 & 2.71 & 1.27 & 4.33 & 32.57 & 28.69 & 77.59 & 52.05 \\
\hline & Pooled & 2.79 & 1.75 & 4.73 & 33.06 & 29.18 & 77.91 & 53.06 \\
\hline \multirow{6}{*}{$\begin{array}{l}\text { No. of Productive } \\
\text { tillers per plant } \\
\text { (PT) }\end{array}$} & E1 & 2.36 & 1.40 & 3.80 & 32.50 & 28.74 & 78.20 & 52.35 \\
\hline & E2 & 2.40 & 1.20 & 3.93 & 29.93 & 26.79 & 80.12 & 49.40 \\
\hline & E3 & 2.45 & 1.53 & 3.67 & 26.67 & 25.20 & 89.28 & 49.05 \\
\hline & E4 & 2.35 & 1.20 & 3.93 & 26.56 & 21.89 & 67.93 & 37.16 \\
\hline & E5 & 2.45 & 1.27 & 3.80 & 26.99 & 25.52 & 89.40 & 49.70 \\
\hline & Pooled & 2.40 & 1.41 & 3.81 & 27.48 & 26.01 & 89.59 & 50.72 \\
\hline \multirow{4}{*}{$\begin{array}{l}\text { No. of leaves per } \\
\text { plant (LP) }\end{array}$} & E1 & 8.41 & 5.67 & 12.20 & 22.61 & 21.46 & 90.09 & 41.96 \\
\hline & E2 & 8.42 & 5.93 & 12.07 & 25.25 & 23.77 & 88.62 & 46.10 \\
\hline & E3 & 8.46 & 5.60 & 12.53 & 21.89 & 20.61 & 88.65 & 39.97 \\
\hline & E4 & 8.38 & 5.73 & 12.23 & 23.82 & 21.32 & 80.11 & 39.31 \\
\hline
\end{tabular}




\begin{tabular}{|c|c|c|c|c|c|c|c|c|}
\hline & E5 & 8.50 & 5.60 & 11.33 & 26.21 & 24.66 & 88.52 & 47.80 \\
\hline & Pooled & 8.43 & 5.72 & 12.01 & 26.71 & 25.16 & 88.73 & 48.82 \\
\hline \multirow{6}{*}{$\begin{array}{l}\text { Spike length (SL) } \\
\text { (cm) }\end{array}$} & E1 & 26.75 & 19.54 & 40.37 & 11.77 & 11.09 & 88.78 & 21.53 \\
\hline & E2 & 26.06 & 18.42 & 37.09 & 13.46 & 13.02 & 93.57 & 25.94 \\
\hline & E3 & 27.54 & 20.07 & 37.36 & 14.36 & 13.24 & 85.01 & 25.15 \\
\hline & E4 & 26.57 & 18.17 & 41.43 & 12.89 & 11.63 & 81.41 & 21.62 \\
\hline & E5 & 27.23 & 19.04 & 41.20 & 14.68 & 13.56 & 85.32 & 25.79 \\
\hline & Pooled & 26.83 & 20.06 & 39.49 & 15.17 & 14.05 & 85.78 & 26.81 \\
\hline \multirow{6}{*}{$\begin{array}{l}\text { Spike girth (SG) } \\
(\mathrm{cm})\end{array}$} & E1 & 1.82 & 1.21 & 2.53 & 31.79 & 30.51 & 92.11 & 60.32 \\
\hline & E2 & 1.85 & 1.28 & 2.77 & 31.70 & 29.55 & 86.90 & 56.74 \\
\hline & E3 & 1.82 & 1.19 & 2.61 & 30.09 & 29.75 & 97.75 & 60.59 \\
\hline & E4 & 1.85 & 1.19 & 2.75 & 31.05 & 30.93 & 99.23 & 63.47 \\
\hline & E5 & 1.84 & 1.19 & 2.54 & 30.41 & 30.07 & 97.78 & 61.24 \\
\hline & Pooled & 1.84 & 1.21 & 2.60 & 30.90 & 30.56 & 97.81 & 62.27 \\
\hline \multirow{6}{*}{$\begin{array}{l}\text { Ear exertion } \\
\text { distance (EED) } \\
(\mathrm{cm})\end{array}$} & E1 & 6.13 & 1.47 & 11.33 & 23.27 & 22.96 & 97.35 & 46.67 \\
\hline & E2 & 6.18 & 1.71 & 11.00 & 25.51 & 25.18 & 97.43 & 51.20 \\
\hline & E3 & 6.02 & 1.49 & 11.54 & 24.02 & 23.71 & 97.44 & 48.21 \\
\hline & E4 & 5.98 & 1.57 & 10.70 & 24.09 & 23.96 & 98.92 & 49.09 \\
\hline & E5 & 6.15 & 1.57 & 11.59 & 24.34 & 24.03 & 97.47 & 48.86 \\
\hline & Pooled & 6.09 & 1.56 & 11.04 & 24.83 & 24.52 & 97.52 & 49.89 \\
\hline \multirow{6}{*}{$\begin{array}{l}\text { Test weight (TW) } \\
\text { (g) }\end{array}$} & E1 & 8.16 & 5.93 & 10.09 & 21.04 & 20.06 & 90.90 & 39.40 \\
\hline & E2 & 8.01 & 5.80 & 10.15 & 32.54 & 29.52 & 82.30 & 55.17 \\
\hline & E3 & 8.07 & 5.83 & 10.02 & 34.93 & 32.55 & 86.84 & 62.48 \\
\hline & E4 & 8.09 & 4.86 & 10.04 & 32.29 & 29.43 & 83.07 & 55.26 \\
\hline & E5 & 8.09 & 5.87 & 9.96 & 35.25 & 32.87 & 86.95 & 63.13 \\
\hline & Pooled & 8.08 & 5.84 & 9.90 & 35.74 & 33.36 & 87.13 & 64.15 \\
\hline \multirow{6}{*}{$\begin{array}{l}\text { Yield per plant } \\
(\text { YP) }(\mathrm{cm})\end{array}$} & E1 & 101.77 & 25.82 & 220.79 & 26.17 & 25.37 & 93.98 & 50.66 \\
\hline & E2 & 110.77 & 25.97 & 255.47 & 26.31 & 24.81 & 88.92 & 48.19 \\
\hline & E3 & 113.72 & 32.89 & 275.29 & 25.15 & 24.37 & 93.89 & 48.65 \\
\hline & E4 & 114.34 & 32.57 & 265.56 & 25.17 & 24.95 & 98.26 & 50.95 \\
\hline & E5 & 109.32 & 31.36 & 272.40 & 25.47 & 24.69 & 93.97 & 49.29 \\
\hline & Pooled & 109.98 & 31.24 & 256.27 & 25.96 & 25.18 & 94.08 & 50.32 \\
\hline \multirow{6}{*}{$\begin{array}{l}\text { Stover yield per } \\
\text { plant (SY) (g) }\end{array}$} & E1 & 490.09 & 291.95 & 772.06 & 11.08 & 9.91 & 80.00 & 18.26 \\
\hline & E2 & 468.70 & 232.65 & 741.33 & 13.46 & 10.04 & 55.64 & 15.43 \\
\hline & E3 & 452.23 & 248.25 & 694.31 & 10.74 & 9.58 & 79.57 & 17.60 \\
\hline & E4 & 473.27 & 242.00 & 768.24 & 9.17 & 8.95 & 95.26 & 17.99 \\
\hline & E5 & 463.68 & 237.48 & 769.33 & 10.06 & 9.40 & 87.30 & 18.08 \\
\hline & Pooled & 469.59 & 281.54 & 708.38 & 10.55 & 10.39 & 96.99 & 21.09 \\
\hline \multirow{6}{*}{$\begin{array}{l}\text { Harvest index (HI) } \\
(\%)\end{array}$} & E1 & 16.55 & 7.22 & 34.89 & 11.08 & 10.72 & 93.61 & 21.37 \\
\hline & E2 & 18.45 & 6.08 & 38.93 & 11.81 & 11.36 & 92.52 & 22.51 \\
\hline & E3 & 19.30 & 8.24 & 37.50 & 12.74 & 10.35 & 66.00 & 17.32 \\
\hline & E4 & 19.14 & 9.55 & 46.71 & 11.81 & 9.48 & 64.43 & 15.68 \\
\hline & E5 & 18.51 & 7.15 & 37.34 & 12.06 & 10.67 & 78.27 & 19.44 \\
\hline & Pooled & 18.39 & 8.86 & 39.07 & 11.55 & 11.16 & 93.36 & 22.22 \\
\hline
\end{tabular}


Table.2 Genotypic (above diagonal) and phenotypic (below diagonal) correlation of different traits in E1 (kharif 2012) and E2 (kharif 2013)

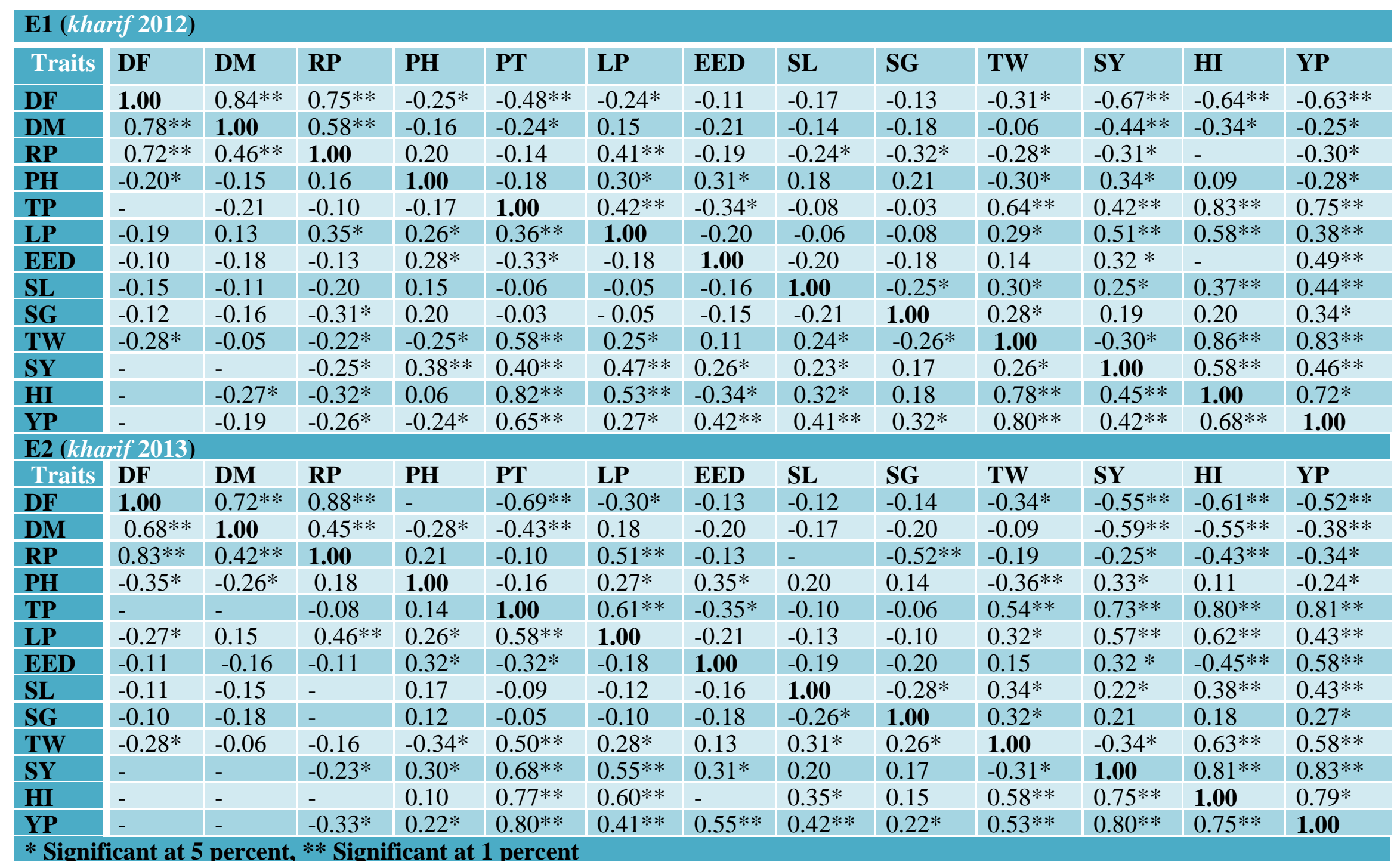


Table.3 Genotypic (above diagonal) and phenotypic (below diagonal) correlation of different traits in E3 (kharif 2014) and E4 (kharif 2015)

\begin{tabular}{|c|c|c|c|c|c|c|c|c|c|c|c|c|c|}
\hline \multicolumn{14}{|c|}{ E3 (kharif 2014) } \\
\hline Traits & DF & DM & $\mathbf{R P}$ & PH & TP & LP & EED & SL & SG & TW & SY & HI & YP \\
\hline DF & 1.00 & $0.63 * *$ & $0.78 * *$ & $-0.30 *$ & $-0.50 * *$ & $-0.28 *$ & -0.20 & -0.12 & -0.14 & $-0.36 * *$ & $-0.73 * *$ & $-0.80 * *$ & $-0.78 * *$ \\
\hline DM & $0.61 * *$ & 1.00 & $0.53 * *$ & -0.21 & $-0.23 *$ & 0.09 & -0.18 & -0.13 & -0.11 & -0.12 & $-0.58 * *$ & $-0.49 * *$ & $-0.37 * *$ \\
\hline $\mathbf{R P}$ & $0.74 * *$ & $0.51 * *$ & 1.00 & 0.14 & -0.20 & $0.40 * *$ & -0.19 & $-0.23^{*}$ & $-0.35^{*}$ & $-0.32 *$ & $-0.25^{*}$ & $-0.47 * *$ & $-0.25^{*}$ \\
\hline PH & $-0.25^{*}$ & -0.18 & 0.13 & 1.00 & -0.21 & $0.25^{*}$ & $0.35^{*}$ & 0.14 & 0.18 & $-0.24 *$ & $0.31 *$ & 0.10 & $-0.33^{*}$ \\
\hline TP & $-0.46^{* *}$ & $-0.22 *$ & -0.18 & 0.20 & 1.00 & $0.45^{* *}$ & $-0.37 * *$ & -0.13 & -0.09 & $0.68 * *$ & $0.49 * *$ & $0.75^{* *}$ & $0.71 * *$ \\
\hline $\mathbf{L P}$ & $-0.25^{*}$ & 0.08 & $0.36^{* *}$ & $-0.24 *$ & $0.41 * *$ & 1.00 & 0.14 & -0.10 & -0.08 & $0.30 *$ & $0.52 * *$ & $0.61 * *$ & $0.44 * *$ \\
\hline EED & -0.18 & -0.15 & -0.16 & $0.33 *$ & $-0.35 *$ & 0.13 & 1.00 & -0.21 & -0.16 & 0.15 & $-0.35^{*}$ & $-0.43 * *$ & $0.52 * *$ \\
\hline SL & -0.11 & -0.13 & $-0.22 *$ & 0.12 & -0.12 & -0.08 & -0.20 & 1.00 & $-0.25^{*}$ & $0.30 *$ & $0.25^{*}$ & $0.37 * *$ & $0.43 * *$ \\
\hline SG & -0.13 & -0.09 & $-0.30 *$ & 0.15 & -0.08 & -0.06 & -0.13 & $-0.22^{*}$ & 1.00 & $0.30 *$ & 0.15 & 0.13 & $0.32 *$ \\
\hline TW & $-0.32 *$ & -0.11 & $-0.28^{*}$ & $-0.22 *$ & $0.61 * *$ & $0.27 *$ & 0.14 & $0.27 *$ & $0.28 *$ & 1.00 & $-0.34 *$ & $0.75^{* *}$ & $0.72 * *$ \\
\hline SY & $-0.69 * *$ & $-0.57 * *$ & $-0.24^{*}$ & $0.30 *$ & $0.43 * *$ & $0.50 * *$ & $-0.32 *$ & $0.24 *$ & 0.11 & $-0.33^{*}$ & 1.00 & $0.68 * *$ & $0.70 * *$ \\
\hline HI & $-0.75 * *$ & $-0.43 * *$ & $-0.45 * *$ & 0.09 & $0.74 * *$ & $0.57 * *$ & $-0.38 * *$ & $0.35^{*}$ & 0.12 & $0.72 * *$ & $0.65 * *$ & 1.00 & $0.76^{*}$ \\
\hline YP & $-0.73 * *$ & $-0.34^{*}$ & $-0.24^{*}$ & $-0.31 *$ & $0.68 * *$ & $0.39 * *$ & $0.50 * *$ & $0.39 * *$ & $0.28 *$ & $0.68^{* *}$ & $0.67 * *$ & $0.74 * *$ & 1.00 \\
\hline \multicolumn{14}{|c|}{ E4 (kharif 2015) } \\
\hline Traits & DF & DM & $\mathbf{R P}$ & $\mathbf{P H}$ & TP & LP & EED & SL & SG & TW & SY & HI & YP \\
\hline DF & 1.00 & $0.53 * *$ & $0.62 * *$ & $-0.34 *$ & $-0.53 * *$ & $-0.23 *$ & -0.16 & -0.11 & -0.15 & $-0.38 * *$ & $-0.65 * *$ & $-0.73 * *$ & $-0.75 * *$ \\
\hline DM & $0.51 * *$ & 1.00 & $0.51 * *$ & $-0.23^{*}$ & -0.20 & 0.14 & -0.13 & -0.15 & -0.10 & -0.15 & $-0.54 * *$ & $-0.52 * *$ & $-0.41 *$ \\
\hline $\mathbf{R P}$ & $0.58 * *$ & $0.48 * *$ & 1.00 & 0.15 & -0.21 & $0.36^{* *}$ & $-0.24 *$ & $-0.26^{*}$ & $-0.33^{*}$ & $-0.35^{*}$ & $-0.28 *$ & $-0.57 * *$ & $-0.26^{*}$ \\
\hline PH & $-0.32 *$ & -0.20 & 0.14 & 1.00 & -0.16 & $0.27 *$ & $0.30 *$ & 0.16 & 0.20 & $-0.22 *$ & $0.36 * *$ & 0.18 & $-0.45 * *$ \\
\hline TP & $-0.48 * *$ & -0.18 & 0.20 & -0.14 & 1.00 & $0.58 * *$ & $-0.35^{*}$ & -0.06 & -0.08 & $0.73 * *$ & $0.58 * *$ & $0.83 * *$ & $0.82 * *$ \\
\hline LP & -0.21 & 0.13 & $0.34 *$ & $0.24 *$ & $0.55 * *$ & 1.00 & 0.18 & -0.04 & -0.02 & $0.38 * *$ & $0.61 * *$ & $0.65 * *$ & $0.53 * *$ \\
\hline EED & -0.14 & -0.11 & $0.22 *$ & $0.28 *$ & $-0.32 *$ & 0.16 & 1.00 & -0.20 & -0.15 & 0.13 & $0.32 *$ & $-0.47 * *$ & $0.52 * *$ \\
\hline SL & -0.10 & -0.14 & $0.23 *$ & 0.13 & -0.06 & -0.03 & -0.17 & 1.00 & $-0.24^{*}$ & $0.35^{*}$ & $0.36^{* *}$ & $0.49 * *$ & $0.55^{* *}$ \\
\hline SG & -0.13 & -0.08 & $0.30 *$ & 0.17 & -0.05 & -0.02 & -0.13 & -0.21 & 1.00 & $0.38^{* *}$ & 0.21 & 0.12 & $0.34^{*}$ \\
\hline TW & $-0.27 *$ & -0.14 & $0.31 *$ & $-0.21^{*}$ & $0.71 * *$ & $0.35^{*}$ & 0.11 & $0.33^{*}$ & $0.36 * *$ & 1.00 & $-0.36 * *$ & $0.65 * *$ & $0.70 * *$ \\
\hline SY & $-0.63 * *$ & $-0.51 * *$ & $0.25 *$ & $0.33^{*}$ & $0.54 * *$ & $0.57 * *$ & $0.28 *$ & $0.32 *$ & 0.20 & $-0.31 *$ & 1.00 & $0.63 * *$ & $0.65 * *$ \\
\hline HI & $-0.72 * *$ & $-0.47 * *$ & $0.53 * *$ & 0.16 & $0.80 * *$ & $0.60 * *$ & $-0.45^{* *}$ & $0.40 * *$ & 0.11 & $0.58^{* *}$ & $0.57 * *$ & 1.00 & $0.80 * *$ \\
\hline YP & $-0.73 * *$ & $-0.36 * *$ & $0.23 * *$ & - & $0.78 * *$ & $0.48 * *$ & $0.50 * *$ & $0.52 * *$ & $0.33^{*}$ & $0.67 * *$ & $0.60 * *$ & $0.78 * *$ & 1.00 \\
\hline
\end{tabular}


Table.4 Genotypic (above diagonal) and phenotypic (below diagonal) correlation of different traits in E5 (kharif 2016) and pooled environments

\begin{tabular}{|c|c|c|c|c|c|c|c|c|c|c|c|c|c|}
\hline \multicolumn{14}{|c|}{ E5 (kharif 2016) } \\
\hline Traits & DF & DM & $\mathbf{R P}$ & PH & $\mathbf{T P}$ & $\mathbf{L P}$ & EED & SL & SG & TW & SY & HI & YP \\
\hline DF & 1.00 & $0.53 * *$ & $0.48 * *$ & $-0.26^{*}$ & - & $-0.34 *$ & -0.13 & -0.12 & -0.16 & $-0.54 *$ & $-0.54 * *$ & $-0.62 * *$ & $-0.65 * *$ \\
\hline DM & $0.51 * *$ & 1.00 & $0.57 * *$ & -0.20 & $-0.26^{*}$ & 0.20 & -0.15 & -0.18 & -0.13 & -0.14 & $-0.51 * *$ & $-0.57 *$ & $-0.41 *$ \\
\hline $\mathbf{R P}$ & $0.45^{* *}$ & $0.53 * *$ & 1.00 & -0.18 & -0.20 & $0.41 * *$ & -0.20 & $-0.34^{*}$ & $-0.28 *$ & $-0.38 * *$ & $-0.30 *$ & $-0.55^{* *}$ & -0.28 \\
\hline PH & $-0.22 *$ & -0.17 & -0.16 & 1.00 & -0.15 & $0.25^{*}$ & $0.31 *$ & 0.19 & 0.11 & $-0.29 *$ & $0.42 * *$ & 0.20 & $-0.45 * *$ \\
\hline TP & $-0.56^{* *}$ & $-0.23^{*}$ & -0.19 & -0.11 & 1.00 & $0.51 * *$ & $-0.32 *$ & -0.10 & -0.10 & $0.65^{* *}$ & $-0.61 * *$ & $0.78 * *$ & $0.80 * *$ \\
\hline $\mathbf{L P}$ & $-0.27 *$ & 0.18 & $0.35 *$ & $0.23 *$ & $0.43 * *$ & 1.00 & $-0.28 *$ & -0.09 & -0.09 & $0.51 * *$ & $0.73 * *$ & $0.57 * *$ & $0.68 * *$ \\
\hline EED & -0.11 & -0.13 & -0.15 & $0.26^{*}$ & $-0.28 *$ & $-0.23 *$ & 1.00 & -0.21 & -0.20 & 0.21 & $0.36^{* *}$ & $-0.52 * *$ & $0.49 * *$ \\
\hline SL & -0.10 & -0.15 & $-0.29^{*}$ & 0.16 & -0.09 & -0.06 & -0.20 & 1.00 & $-0.34 *$ & $0.30 *$ & $0.45^{* *}$ & $0.58 * *$ & $0.62 * *$ \\
\hline SG & -0.13 & -0.12 & $-0.23^{*}$ & 0.10 & -0.08 & -0.05 & -0.17 & $-0.32^{*}$ & 1.00 & $0.40 * *$ & 0.18 & 0.14 & $0.32 *$ \\
\hline TW & $-0.48 * *$ & -0.11 & $-0.34^{*}$ & $-0.23^{*}$ & $0.50 * *$ & $0.43 * *$ & 0.18 & 0.21 & $0.31 *$ & 1.00 & - & $0.55^{* *}$ & $0.62 * *$ \\
\hline SY & - & - & $-0.27 *$ & $0.40 * *$ & - & $0.65^{* *}$ & $0.31 *$ & $0.35 *$ & 0.15 & $-0.33 *$ & 1.00 & $0.58 * *$ & $0.57 * *$ \\
\hline HI & $-0.57 * *$ & - & - & 0.17 & $0.63 * *$ & $0.51 * *$ & - & $0.43 * *$ & 0.11 & $0.46 * *$ & $0.42 * *$ & 1.00 & $0.68 * *$ \\
\hline YP & $-0.61 * *$ & - & $-0.22 *$ & - & $0.72 * *$ & $0.62 * *$ & $0.38 * *$ & $0.55 * *$ & $0.30 *$ & $0.54 * *$ & $0.53 * *$ & $0.58 * *$ & 1.00 \\
\hline \multicolumn{14}{|l|}{ Pooled } \\
\hline Traits & DF & DM & $\mathbf{R P}$ & PH & TP & LP & EED & SL & SG & TW & SY & HI & YP \\
\hline DF & 1.00 & $0.64 * *$ & $0.57 * *$ & $-0.32 *$ & - & $-0.32 *$ & -0.21 & -0.18 & -0.13 & $-0.34 *$ & $-0.54 * *$ & $-0.59 * *$ & $-0.62 * *$ \\
\hline DM & $0.57 * *$ & 1.00 & $0.54 * *$ & -0.18 & $-0.29 *$ & 0.20 & -0.17 & -0.16 & -0.20 & -0.13 & $-0.48 * *$ & $-0.42 * *$ & $-0.28 *$ \\
\hline $\mathbf{R P}$ & $0.49 * *$ & $0.45^{* *}$ & 1.00 & -0.21 & $-0.25^{*}$ & $0.53 * *$ & -0.20 & $-0.31 *$ & $-0.35^{*}$ & $-0.25^{*}$ & $-0.30 *$ & $-0.53 * *$ & $-0.35^{*}$ \\
\hline PH & $-0.30 *$ & -0.15 & -0.17 & 1.00 & -0.14 & $0.34 *$ & $0.32 *$ & 0.15 & 0.20 & $-0.34 *$ & $0.38 * *$ & 0.18 & $-0.36 * *$ \\
\hline TP & $-0.48 * *$ & $-0.23^{*}$ & $-0.23^{*}$ & -0.11 & 1.00 & $0.61 * *$ & $-0.34 *$ & -0.18 & -0.13 & $0.57 * *$ & $-0.45^{* *}$ & $0.75 * *$ & $0.72 * *$ \\
\hline LP & $-0.25^{*}$ & 0.18 & $0.48 * *$ & $0.32 *$ & $0.57 * *$ & 1.00 & -0.14 & -0.10 & -0.11 & $0.40 * *$ & $0.67 * *$ & $0.64 * *$ & $0.56 * *$ \\
\hline EED & -0.18 & -0.15 & -0.16 & $0.29 *$ & $-0.31 *$ & -0.11 & 1.00 & -0.20 & -0.21 & 0.18 & $0.34^{*}$ & $-0.58 * *$ & $0.67 * *$ \\
\hline SL & -0.16 & -0.13 & $-0.23^{*}$ & 0.13 & -0.16 & -0.08 & -0.18 & 1.00 & $-0.31 *$ & $0.35^{*}$ & $0.22 *$ & $0.54 * *$ & $0.51 * *$ \\
\hline SG & -0.11 & -0.18 & $-0.28 *$ & 0.19 & -0.12 & -0.09 & -0.20 & $-0.27^{*}$ & 1.00 & $0.24 *$ & 0.15 & 0.18 & $0.32 *$ \\
\hline TW & $-0.29 *$ & -0.11 & -0.21 & $-0.28^{*}$ & $0.53^{* *}$ & $0.32 *$ & 0.15 & $0.31 *$ & $0.22 *$ & 1.00 & $-0.33^{*}$ & $0.75^{* *}$ & $0.78^{* *}$ \\
\hline SY & $-0.47 * *$ & - & $-0.29 *$ & $0.34 *$ & - & $0.63 * *$ & $0.32 *$ & 0.19 & 0.13 & $-0.28 *$ & 1.00 & $0.63 * *$ & $0.70 * *$ \\
\hline HI & $-0.53 * *$ & - & - & 0.15 & $0.68 * *$ & $0.61 * *$ & - & $0.51 * *$ & 0.14 & $0.71 * *$ & $0.61 * *$ & 1.00 & $0.68 *$ \\
\hline YP & $-0.59 * *$ & $-0.25^{*}$ & $-0.32 *$ & $-0.33^{*}$ & $0.70 * *$ & $0.54 * *$ & $0.63 * *$ & $0.49 * *$ & $0.30 *$ & $0.68 * *$ & $0.65^{* *}$ & $0.62 * *$ & 1.00 \\
\hline
\end{tabular}


Table.5 The direct (diagonal values in bold) and indirect effects of component traits on yield per plant in E1 and E2

\begin{tabular}{|c|c|c|c|c|c|c|c|c|c|c|c|c|c|}
\hline \multicolumn{14}{|c|}{ E1 (kharif 2012) } \\
\hline Traits & DF & DM & $\mathbf{R P}$ & PH & TP & $\mathbf{L P}$ & EED & SL & SG & TW & SY & HI & Corr. with YP \\
\hline DF & -0.163 & -0.018 & -0.015 & -0.013 & - & 0.026 & -0.022 & 0.027 & 0.021 & - & 0.024 & -0.023 & $-0.63 * *$ \\
\hline DM & 0.129 & -0.116 & -0.014 & -0.022 & - & 0.011 & -0.025 & 0.023 & 0.023 & - & 0.022 & -0.026 & $-0.25^{*}$ \\
\hline $\mathbf{R P}$ & 0.052 & -0.095 & -0.135 & -0.001 & - & 0.023 & -0.027 & 0.018 & 0.025 & - & 0.017 & 0.025 & $-0.30^{*}$ \\
\hline PH & -0.013 & -0.014 & -0.009 & 0.685 & - & 0.027 & -0.071 & 0.032 & 0.027 & - & 0.035 & 0.077 & $-0.28 *$ \\
\hline TP & -0.017 & -0.026 & -0.021 & 0.021 & 0.458 & 0.032 & -0.022 & 0.095 & 0.074 & 0.054 & 0.062 & 0.035 & $0.75 * *$ \\
\hline LP & -0.092 & -0.012 & -0.013 & -0.007 & 0.345 & 0.061 & -0.061 & 0.037 & 0.065 & 0.028 & 0.050 & 0.016 & $0.38 * *$ \\
\hline EED & -0.015 & -0.015 & -0.006 & -0.008 & - & 0.041 & -0.092 & 0.055 & 0.045 & - & 0.044 & 0.063 & $0.49 * *$ \\
\hline SL & -0.036 & -0.015 & -0.021 & -0.013 & 0.138 & 0.037 & 0.011 & 0.531 & 0.018 & - & 0.058 & 0.011 & $0.44 * *$ \\
\hline SG & -0.045 & -0.016 & -0.009 & -0.021 & 0.130 & 0.018 & -0.060 & 0.031 & 0.735 & 0.036 & 0.042 & 0.028 & $0.34 *$ \\
\hline TW & -0.015 & -0.008 & -0.010 & -0.006 & 0.115 & -0.023 & 0.082 & 0.062 & 0.013 & 0.104 & 0.033 & 0.019 & $0.83 * *$ \\
\hline SY & -0.017 & -0.096 & -0.011 & -0.002 & 0.153 & 0.016 & -0.063 & 0.035 & 0.015 & 0.073 & 0.082 & 0.027 & $0.46 * *$ \\
\hline HI & -0.032 & -0.011 & -0.103 & -0.023 & 0.074 & 0.039 & -0.075 & 0.067 & 0.032 & 0.085 & 0.056 & 0.091 & $0.72 *$ \\
\hline \multicolumn{14}{|c|}{ E2 (kharif 2013) } \\
\hline Traits & DF & DM & RP & $\mathbf{P H}$ & TP & $\mathbf{L P}$ & EED & SL & SG & TW & SY & HI & Corr. with YP \\
\hline DF & -0.157 & -0.013 & -0.011 & -0.012 & - & 0.031 & -0.025 & 0.028 & 0.023 & - & 0.026 & -0.018 & $-0.52 * *$ \\
\hline DM & 0.137 & -0.132 & -0.098 & -0.023 & - & 0.023 & -0.022 & 0.023 & 0.020 & - & 0.029 & -0.024 & $-0.38 * *$ \\
\hline RP & 0.049 & -0.043 & -0.106 & -0.006 & - & 0.030 & -0.031 & 0.031 & 0.022 & - & 0.028 & 0.021 & $-0.34 *$ \\
\hline PH & -0.011 & -0.023 & -0.006 & 0.632 & - & 0.025 & -0.068 & 0.045 & 0.025 & - & 0.032 & 0.063 & $-0.24 *$ \\
\hline TP & -0.014 & -0.025 & -0.026 & 0.019 & 0.763 & 0.018 & -0.055 & 0.095 & 0.075 & 0.073 & 0.075 & 0.052 & $0.81^{* *}$ \\
\hline LP & -0.075 & -0.018 & -0.021 & -0.005 & 0.238 & 0.032 & -0.063 & 0.032 & 0.063 & 0.035 & 0.073 & 0.035 & $0.43 * *$ \\
\hline EED & -0.019 & -0.011 & -0.010 & -0.010 & - & 0.025 & -0.079 & 0.059 & 0.048 & - & 0.044 & 0.063 & $0.58 * *$ \\
\hline SL & -0.043 & -0.011 & -0.025 & -0.009 & 0.152 & 0.031 & 0.054 & 0.732 & 0.014 & - & 0.064 & 0.036 & $0.43 * *$ \\
\hline SG & -0.053 & -0.022 & -0.006 & -0.015 & 0.184 & 0.020 & -0.057 & 0.025 & 0.709 & 0.052 & 0.052 & 0.045 & $0.27 *$ \\
\hline TW & -0.024 & -0.010 & -0.015 & -0.011 & 0.135 & -0.022 & 0.064 & 0.071 & 0.023 & 0.635 & 0.038 & 0.023 & $0.58 * *$ \\
\hline SY & -0.010 & -0.065 & -0.021 & -0.009 & 0.173 & 0.031 & -0.072 & 0.062 & 0.034 & 0.072 & 0.132 & 0.043 & $0.83 * *$ \\
\hline HI & -0.043 & -0.013 & -0.232 & -0.018 & 0.112 & 0.032 & -0.075 & 0.059 & 0.038 & 0.095 & 0.058 & 0.082 & $0.79 *$ \\
\hline
\end{tabular}


Table.6 The direct (diagonal values in bold) and indirect effects of component traits on yield per plant in E3 and E4

\begin{tabular}{|c|c|c|c|c|c|c|c|c|c|c|c|c|c|}
\hline \multicolumn{14}{|c|}{ E3 (kharif 2013) } \\
\hline Traits & DF & DM & $\mathbf{R P}$ & PH & TP & LP & EED & SL & SG & TW & SY & HI & Corr. with YP \\
\hline DF & -0.244 & -0.012 & -0.022 & -0.011 & - & 0.018 & -0.023 & 0.033 & 0.025 & - & 0.023 & -0.025 & $-0.78 * *$ \\
\hline DM & 0.154 & -0.213 & -0.022 & -0.023 & - & 0.013 & -0.021 & 0.026 & 0.029 & - & 0.032 & -0.028 & $-0.37 * *$ \\
\hline RP & 0.112 & -0.063 & -0.152 & -0.012 & - & 0.022 & -0.032 & 0.021 & 0.023 & - & 0.021 & 0.026 & $-0.25^{*}$ \\
\hline PH & -0.016 & -0.022 & -0.032 & 0.425 & - & 0.025 & -0.063 & 0.035 & 0.025 & - & 0.042 & 0.048 & $-0.33^{*}$ \\
\hline TP & -0.015 & -0.032 & -0.025 & 0.035 & 0.538 & 0.036 & -0.035 & 0.083 & 0.072 & 0.050 & 0.032 & 0.037 & $0.71 * *$ \\
\hline LP & -0.102 & -0.018 & -0.011 & -0.012 & 0.286 & 0.125 & -0.064 & 0.058 & 0.054 & 0.032 & 0.037 & 0.049 & $0.44 * *$ \\
\hline EED & -0.011 & -0.012 & -0.013 & -0.010 & - & 0.045 & -0.065 & 0.083 & 0.061 & - & 0.043 & 0.036 & $0.52 * *$ \\
\hline SL & -0.054 & -0.011 & -0.022 & -0.019 & 0.210 & 0.032 & 0.034 & 0.643 & 0.016 & - & 0.038 & 0.045 & $0.43 * *$ \\
\hline SG & -0.032 & -0.022 & -0.015 & -0.032 & 0.152 & 0.021 & -0.061 & 0.057 & 0.685 & 0.035 & 0.035 & 0.033 & $0.32 *$ \\
\hline TW & -0.026 & -0.010 & -0.010 & -0.013 & 0.113 & -0.018 & 0.055 & 0.065 & - & 0.355 & 0.036 & 0.029 & $0.72 * *$ \\
\hline SY & -0.021 & -0.062 & -0.026 & -0.011 & 0.162 & 0.015 & -0.054 & 0.048 & 0.034 & 0.056 & 0.046 & 0.023 & $0.70 * *$ \\
\hline HI & -0.042 & -0.042 & -0.182 & -0.032 & 0.043 & 0.033 & -0.045 & 0.083 & 0.031 & 0.064 & - & 0.051 & $0.76^{*}$ \\
\hline \multicolumn{14}{|c|}{ E4 (kharif 2016) } \\
\hline Traits & DF & DM & $\mathbf{R P}$ & PH & $\mathbf{T P}$ & LP & EED & SL & SG & TW & SY & HI & Corr. with YP \\
\hline DF & -0.252 & -0.011 & -0.021 & -0.013 & - & 0.013 & -0.021 & - & 0.030 & - & 0.023 & -0.013 & $-0.75^{* *}$ \\
\hline DM & 0.154 & -0.193 & -0.025 & -0.025 & - & 0.016 & -0.025 & - & 0.031 & - & 0.027 & -0.024 & $-0.41 *$ \\
\hline RP & 0.032 & -0.032 & -0.204 & -0.032 & - & 0.030 & -0.023 & - & 0.023 & - & 0.023 & 0.022 & $-0.26^{*}$ \\
\hline PH & -0.013 & -0.028 & -0.045 & 0.536 & - & 0.025 & -0.048 & - & 0.032 & - & 0.064 & 0.075 & $-0.45 * *$ \\
\hline TP & -0.011 & -0.032 & -0.029 & 0.032 & 0.613 & 0.023 & -0.043 & - & 0.098 & 0.069 & 0.062 & 0.081 & $0.82 * *$ \\
\hline LP & -0.082 & -0.021 & -0.032 & -0.023 & 0.211 & 0.042 & -0.043 & - & 0.076 & 0.042 & 0.071 & 0.063 & $0.53 * *$ \\
\hline EED & -0.032 & -0.017 & -0.045 & -0.017 & - & 0.034 & -0.052 & - & 0.073 & - & 0.058 & 0.057 & $0.52 * *$ \\
\hline SL & -0.057 & -0.009 & -0.036 & -0.015 & 0.134 & 0.054 & 0.038 & 0.598 & 0.055 & - & 0.069 & 0.048 & $0.55^{* *}$ \\
\hline SG & -0.062 & -0.028 & -0.013 & -0.023 & 0.215 & 0.032 & -0.033 & 0.043 & 0.674 & 0.064 & 0.073 & 0.067 & $0.34 *$ \\
\hline TW & -0.044 & -0.015 & -0.019 & -0.018 & 0.164 & -0.013 & 0.050 & 0.087 & 0.048 & 0.393 & 0.059 & 0.032 & $0.70 * *$ \\
\hline SY & -0.021 & -0.084 & -0.016 & -0.012 & 0.214 & 0.034 & -0.047 & 0.074 & 0.061 & 0.081 & - & 0.055 & $0.65 * *$ \\
\hline HI & -0.058 & -0.053 & -0.120 & -0.025 & 0.164 & 0.040 & -0.032 & 0.079 & 0.058 & 0.074 & 0.065 & -0.087 & $0.80 * *$ \\
\hline
\end{tabular}


Table.7 The direct (diagonal values in bold) and indirect effects of component traits on yield per plant in E5 and pooled environments

\begin{tabular}{|c|c|c|c|c|c|c|c|c|c|c|c|c|c|}
\hline \multicolumn{14}{|c|}{ E5 (kharif 2016) } \\
\hline Traits & DF & DM & $\mathbf{R P}$ & PH & TP & LP & EED & SL & SG & TW & SY & HI & Corr. with YP \\
\hline DF & 0.143 & -0.015 & -0.013 & -0.021 & -0.024 & 0.026 & - & 0.02 & 0.031 & - & 0.034 & - & $-0.65 * *$ \\
\hline DM & 0.125 & -0.142 & -0.034 & -0.033 & -0.021 & 0.025 & - & 0.02 & 0.026 & - & 0.023 & - & $-0.41 *$ \\
\hline $\mathbf{R P}$ & 0.037 & -0.035 & -0.128 & -0.013 & -0.027 & 0.030 & - & 0.02 & 0.030 & - & 0.028 & 0.019 & -0.28 \\
\hline PH & - & -0.021 & -0.032 & 0.645 & -0.045 & 0.031 & - & 0.06 & 0.058 & - & 0.067 & 0.058 & $-0.45^{* *}$ \\
\hline TP & - & -0.034 & -0.025 & 0.022 & 0.735 & 0.028 & - & 0.09 & 0.066 & 0.073 & 0.075 & 0.063 & $0.80 * *$ \\
\hline $\mathbf{L P}$ & - & -0.020 & -0.032 & -0.010 & 0.205 & 0.057 & - & 0.07 & 0.054 & 0.035 & 0.079 & 0.054 & $0.68 * *$ \\
\hline EED & - & -0.021 & -0.013 & -0.015 & -0.028 & 0.027 & - & 0.06 & 0.057 & - & 0.068 & 0.053 & $0.49 * *$ \\
\hline SL & - & -0.015 & -0.024 & -0.018 & 0.210 & 0.031 & 0.062 & 0.71 & 0.048 & - & 0.066 & 0.032 & $0.62 * *$ \\
\hline SG & - & -0.024 & -0.012 & -0.023 & 0.225 & 0.020 & - & 0.05 & 0.698 & 0.052 & 0.054 & 0.035 & $0.32 *$ \\
\hline TW & - & -0.023 & -0.011 & -0.021 & 0.159 & -0.021 & 0.072 & 0.08 & 0.032 & 0.658 & 0.046 & 0.024 & $0.62 * *$ \\
\hline SY & - & -0.045 & -0.021 & -0.013 & 0.136 & 0.029 & - & 0.07 & 0.027 & 0.072 & 0.157 & 0.047 & $0.57 * *$ \\
\hline HI & - & -0.031 & -0.122 & -0.026 & 0.108 & 0.023 & - & 0.06 & 0.031 & 0.095 & 0.032 & 0.091 & $0.68 * *$ \\
\hline \multicolumn{14}{|c|}{ Pooled Environment } \\
\hline Traits & DF & DM & RP & PH & TP & LP & EED & SL & SG & TW & SY & HI & Corr. with YP \\
\hline DF & 0.148 & -0.011 & -0.024 & -0.018 & -0.022 & 0.019 & - & 0.02 & 0.020 & - & 0.023 & - & $-0.62 * *$ \\
\hline DM & 0.120 & -0.163 & -0.027 & -0.026 & -0.024 & 0.013 & - & 0.02 & 0.024 & - & 0.032 & - & $-0.28 *$ \\
\hline $\mathbf{R P}$ & 0.104 & -0.054 & -0.147 & -0.025 & -0.027 & 0.022 & - & 0.03 & 0.019 & - & 0.021 & 0.026 & $-0.35^{*}$ \\
\hline PH & - & -0.038 & -0.037 & 0.463 & -0.035 & 0.024 & - & 0.03 & 0.023 & - & 0.049 & 0.076 & $-0.36 * *$ \\
\hline TP & - & -0.035 & -0.021 & 0.034 & 0.620 & 0.036 & - & 0.06 & 0.062 & 0.033 & 0.064 & 0.064 & $0.72 * *$ \\
\hline LP & - & -0.017 & -0.018 & -0.028 & 0.237 & 0.125 & - & 0.05 & 0.054 & 0.035 & 0.065 & 0.057 & $0.56^{* *}$ \\
\hline EED & - & -0.022 & -0.014 & -0.019 & -0.048 & 0.035 & - & 0.05 & 0.063 & - & 0.081 & 0.068 & $0.67 * *$ \\
\hline SL & - & -0.015 & -0.027 & -0.025 & 0.176 & 0.031 & 0.061 & 0.63 & 0.023 & - & 0.078 & 0.063 & $0.51 * *$ \\
\hline SG & - & -0.034 & -0.035 & -0.035 & 0.135 & 0.028 & - & 0.05 & 0.654 & 0.035 & 0.065 & 0.058 & $0.32 *$ \\
\hline TW & - & -0.013 & -0.018 & -0.017 & 0.128 & -0.020 & 0.059 & 0.06 & - & 0.383 & 0.063 & 0.049 & $0.78 * *$ \\
\hline SY & - & -0.043 & -0.023 & -0.015 & 0.122 & 0.017 & - & 0.05 & 0.057 & 0.033 & 0.183 & 0.053 & $0.70 * *$ \\
\hline HI & - & -0.031 & -0.093 & -0.023 & 0.065 & 0.032 & - & 0.04 & 0.035 & 0.024 & - & 0.085 & $0.68 *$ \\
\hline
\end{tabular}


Fig.1 Components of variance seed yield per plant and its components traits for five environments in pearl millet

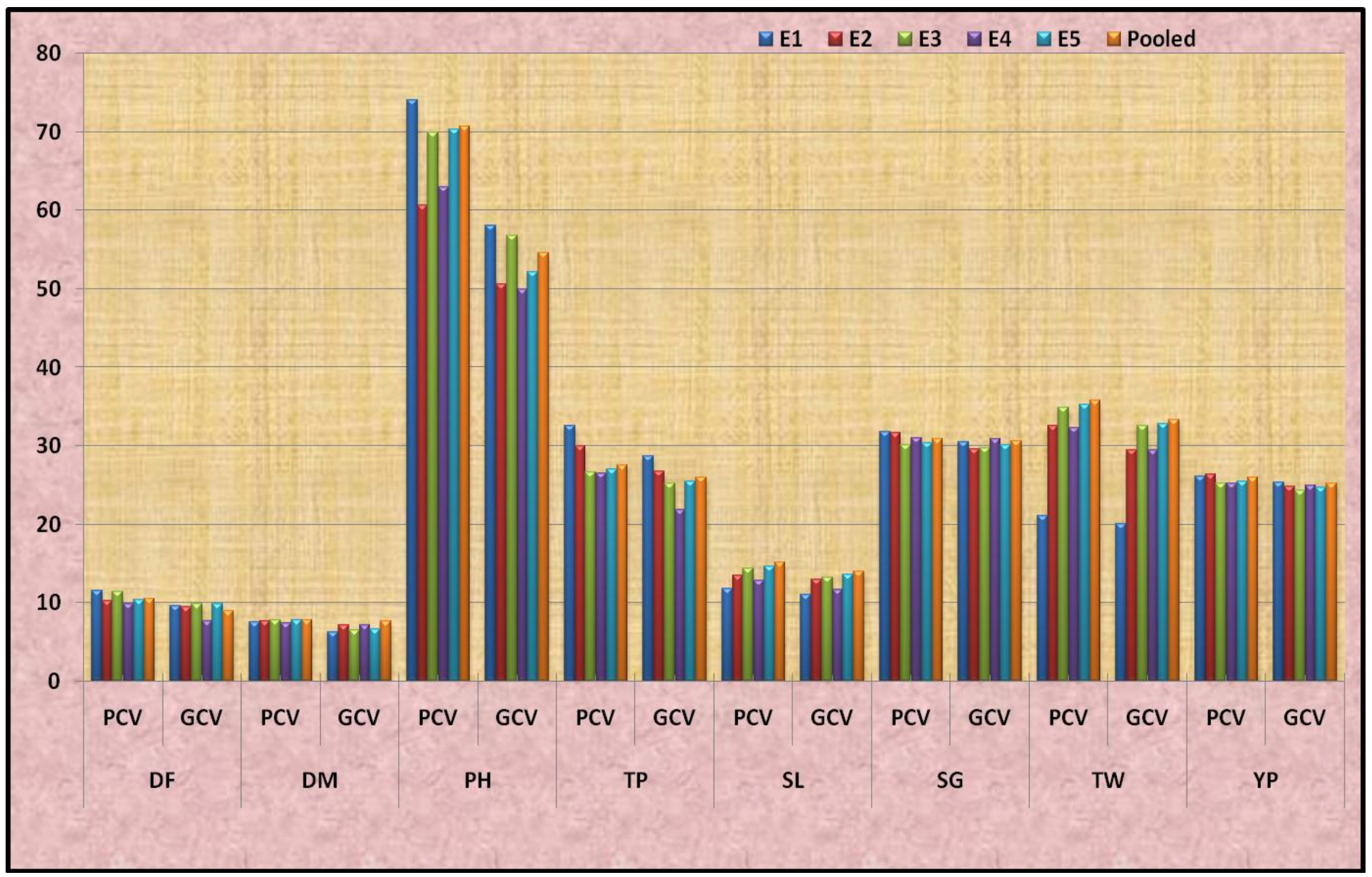


Thus, it indicates that phenotype was fairly good indication of genotype under particular environment and selection on the basis of phenotype should be useful for the attributes under selection (Gupta and Mishra, 2005 and Salini et al., 2010).

High heritability coupled with moderate genetic advance was observed for days to flowering and days to maturity that implies equal importance of additive and non additive gene actions. The estimates of heritability in broad sense and genetic advance for the studied characters were slightly fluctuating at the different seasons. The differences in the magnitude of heritability would be attributed to the effect of the environment. Robinson et al., (1949) attributed the change in heritability estimates in maize (Zea mays L.) to differential response of genotype to the environment.

\section{Correlation and path analysis}

Estimation of correlation coefficients between different pair of traits under study revealed that not all traits are correlated to each other or with seed yield per plant. Considering the correlation between seed yield per plant and other characters, it was found that seed yield was positively and significantly correlated with number of productive tillers per plant, number of leaves per plant, spike length, spike girth, test weight, stover yield and harvest index in all the environments and in pooled environment (Table 2, 3 and 4). Hence, these characters have to be given importance during the selection programme to improve the yield potential of the crop. From the aforesaid facts, it was clear that all the yield component traits viz., days to 50 per cent flowering, days to maturity, reproductive period, plant height, number of productive tillers per plant, number of leaves per plant, ear exertion distance, spike length, spike girth, test weight, stover yield and harvest index were inter correlated among themselves. Kumar et al., (2013) observed significant and positive correlation of grain yield per plant with plant height, number of tillers per plant and test weight in wheat. Similar kind of association was revealed by Aruselvi et al., (2008) and Meena kumara et al., (2008) for 1000-grain weight and Dhakar et al., (2012) reported for panicle diameter.

Among the other traits positive and significant phenotypic and genotypic correlation coefficients were observed for days to fifty percent flowering with days to maturity and reproductive period; days to maturity with reproductive period; reproductive period with number of leaves per plant; plant height with number of leaves per plant, ear exertion distance and stover yield per plant; number of productive tillers per plant with number of leaves per plant, test weight, stover yield per plant and harvest index; number of leaves per plant with test weight, stover yield per plant and harvest index; ear exertion distance trait with stover yield; spike length with test weight, stover yield per plant and harvest index; spike girth with test weight; test weight and stover yield per plant with harvest index.

\section{Path co efficient analysis}

The correlation coefficients between any two characters would not give a complete picture of a complex situation like yield of plant which is jointly determined by a number of traits either directly or indirectly. In such situation, path coefficient analysis would be useful, as it permits the separation of direct effect from indirect effects through other related traits by partitioning the genotypic correlation coefficient (Dewey and Lu, 1959).

The values of path coefficients are presented in Table 5, 6 and 7. The highest positive direct effect registered by spike girth $(0.735)$ 
followed by plant height (0.685), spike length $(0.531)$ and plant height (0.458) in E1, number of productive tiller per plant $(0.763)$ followed by spike length (0.732), spike girth (0.709), test weight (0.635) and plant height (0.632) in E2; spike girth (0.685) followed by spike length (0.643), number of productive tillers per plant (0.538), plant height $(0.425)$ and test weight $(0.355)$ in E3; spike girth (0.673) followed by number of productive tillers per plant (0.613), spike length (0.598), plant height (0.536) and test weight (0.393) in E4; number of productive tillers per plant (0.735) followed by spike length (0.712), spike girth (0.698), plant height (0.645) and test weight (0.658), while in pooled environment highest positive direct effect was recorded by spike girth (0.654) followed by spike length (0.631), number of productive tillers per plant $(0.620)$, plant height $(0.463)$ and test weight $(0.383)$.

These results are in accordance with Vetriventhan and Nirmala kumari (2007), Aruselvi et al., (2008) and Dhakar et al., (2012) for panicle girth; Poornima et al., (2004), Salunke et al., (2006), Vetriventhan and Nirmala kumari (2007), Aruselvi et al., (2008) and Meena kumara et al., (2008). For positive direct effect of panicle length on grain yield per plant; Hepziba et al., (1993), Salunke et al., (2006) and Vetriventhan and Nirmala kumari (2007) produce tiller per plant in pearl millet; Mohan Lal and Maloo (2006) in barnyard millet for positive direct effect of plant height on grain yield per plant; Salunke et al., (2006), Vetriventhan and Nirmala kumari (2007), Aruselvi et al., (2008) and Kumari and Nagarajan (2008) for positive direct effect of 1000-grain weight on grain yield per plant. The negative direct effects on grain yield were exerted by days to fifty percent flowering, days to maturity, reproductive period and ear exertion distance. These results are in agreement with the reports of Salunke et al., (2006), Aruselvi et al., (2008) and Dhakar et al., (2012) for days to 50\% flowering, Salunke et al., (2006) for days to maturity, and days to $50 \%$ flowering. The present study of genotypic path coefficient analysis revealed highest positive direct effect registered by spike girth, spike length, number of productive tillers per plant, plant height and test weight.

\section{References}

Aruselvi S. Mohanasundaram and Selvi B. 2008. Correlation and path analysis for grain yield and its components in pearl millet (Pennisetum glaucum (L.) R. Br.) The Madras Agricultural J 95(7-12): 311-314.

Bhoite K.D., Pardeshi S.R., Mhaske B.M., Wagh M.P. 2008. Study of genetic variability in pearl millet (Pennisetum glaucum L.). Agric Sci Digest 28:111- 117.

Bhuri Singh, Upadhya P.K. and Sharma K.C. 2014. Genetic Variability, Correlation and Path Analysis In Pearl Millet (Pennisetum glaucum (L.) R. Br.). Indian Res J Genet \& Biotech. 6: 491-500.

Burton G.W. 1952. Quantitative inheritance in grasses. Proc. 6th Int. Grassland Cong1: 277-283.

Chaudhary V.R., Dhedhi K.K., Joshi H.J. and Sorathiya J.S. 2012. Character association and path analysis in pearl millet (Pennisetum glaucum L.) Asian Journal of Bio Science 7(1): 98-100.

Dapke J.S., Shah D.S., Pawar G.N., Dhembre V.M. and Mithilesh K. 2014. Genetic variability and character association over environment in pearl millet (Pennisetum glaucum L.) under dry land conditions of Gujarat. The Bioscan 9(2): 863-867.

Deb Choudhary P.K. and Das P.K. 1998. Genetic variability, correlation and path coefficient analysis in deep water rice Ann of Agric. Res 19(2): 120-121.

Dewey D.R. and Lu, K.M. 1959. Correlation and path coefficient analysis of crested wheat grass seed production. Agron J 51: 515516.

Dhakar A.K., Verma P.K., Mittal S.B., Yadav H.P., Devvart and Chugh L.K. 2012. Interrelationships among yields and quality 
parameters in pearl millet hybrids under rain fed conditions Forage Research 38(1): 32-34.

Falconer D.S. 1964. Introduction to quantitative genetics. Oliverand Boyd Ltd., London, W.I. Falconer D S 1981. Introduction to quantitative Genetics. Oliver Boyd, London. 340.

Govindaraj M., Selvi B., Rajarathinam S. and Sumathi P. 2011. Genetic variability and heritability of grain yield components and grain mineral concentration in India's pearl millet (Pennisetum glaucum (L.) R. Br.) accessions. African J Food Agric. Nutr Dev 11: 4758-4771.

Gupta R.R. and Mishra R. 2005. Genetic variability and heritability of yield and its components in finger millet (Eleusine coracana (L.) Geartn.) Madras Agric J 92(1):157-159.

Harinayan B., Baudh Bharti, Sanjeev Kumar, Pandey M.K., Kumar D. and Vishwakarma D.N. 2015. Studies on genetic variability for fodder yield and its contributing characters in bajra (Pennisetum glaucum L.) Agric Sci Digest 35(1): 78-80.

Hepziba S.J., Saraswathi R., Mani M.T., Rajasekaran R. and Palanisamy S. 1993. Genetic variability, association among metric traits and path coefficient analysis in pearl millet Annals of Agricultural Research 14(3): 282-285.

Johnson H.W., Robinson H.F. and Comstock R.E. 1955. Genotypic and phenotypic correlation in soybeans and their implication in selection. Agronomy J 47: 477-483.

Kumar B., Singh C.M. and Jaiswal K.K. 2013. Genetic variability, association and diversity studies in bread wheat (Triticum aestivum L.) The Bioscan 8(1): 143-147.

Kumar R., Harish S., Dalal M.S., Malik V., Devvart L.K., Chugh P., Garg and Raj K. 2014. Studies on variability and character association under rainfed conditions in pearl millet (Pennisetum glaucum L.) hybrids Forage Res 40: 163-167.

Kumari B.M. and Nagarajan P. 2008. Character association and path analysis of yield components in pearl millet (Pennisetum glaucum L.) Madras Agricultural Journal 95(1/6): 192-195.
Lakshmana D., Biradar B.D and Ravi Kumar R.L. 2009. Genetic Variability Studies for Quantitative Traits in a Pool of Restorers and Maintainers Lines of Pearl Millet (Pennisetum glaucum L.). Karnataka J Agric Sci 22(4): 881-882.

Lakshmana D., Biradar B.D., Madaiah D. and Jolli R.B. 2010. Genetic diversity for yield and its component traits in pearl millet germplasm Indian Journal of Plant Genetic Resources 23(2): 206-211.

Lush J.L. 1940. Intra-sire correlation and regression of offspring on dam as a method of estimating heritability of characters Proceedings of American Society for Animal Production 33: 293-301

Majumder M.K., Chatterjee S.D., Bose P. and Bhattacharya G. 1974. Variability, inter relationships and path analysis for some quantitative characters in okra (Abelmoschus esculentus (L.) Moench) Indian Agric18: 13-20

Marwede V., Schierholt A. and Becker H.C. 2004. Genotype $\mathrm{x}$ environment interactions and heritability of tocopherol contents in canola Crop Sci 44: 728-731.

MeenaKumari B. and Nagarajan P. 2008. Variability and Heritability Analysis in pearl millet (Pennisetum glaucum L.)The Madras Agricultural J 95(1-6): 190-192.

Mehdi S.S. and Khan I. A. 1994. Experimental design and analysis. In: Plant Breeding (Eds.): E. Bashir and R. Bantel National Book Foundation Islamabad pp. 215.

Mohan Lal and Maloo S.R. 2006. Path coefficient analysis for seed yield in barnyard millet (Echnichloa frumentacea (Roxb.) Link.) Agric Sci Digest 26(2).

Panse V.G. and Sukhatme P.V. 1967. Statistical methods for agricultural workers 2 nd Ed. ICAR., New Delhi.

Poornima Mangal, Singh A.K., Indapurkar Y.M. and Singhal H.C. 2004. Path and association studies among yield, quantity and physiological components in pearl millet Agriculture Science Digest 24(2): 9598.

Robinson H.F., Comstock R.E., Harvey P.H. 1949. Estimation of Heritability and Degree of Dominance in Corn. Agron. J. 41:353359. 
Salih A.I.S., Mohamed I.I., Elgailani A., Khalid A.O. and Adam M.A. 2014. Genetic variation among pearl millet accessions for yield and its components in semi-arid zone Sudan Intl J Agri Crop Sci 7(11): 822-826.

Salini K., Nirmalakumari A., Muthiah A.R. and Senthil N. 2010. Evaluation of proso millet (Panicum miliaceum L.) germplasm collections Electronic Journal of Plant Breeding, 1(4):489-499.

Salunke P.K., Dumbre A.D. and Rajput S.D. 2006. Correlation and path analysis in germplasms of pearl millet (Pennisetum glaucumL.) Journal of Maharashtra Agricultural University 31(1): 16-18.

Saraswathi R.S., Juliet Hepziba M., Theradi Mani, Palanisamy S. and AKFazlullah Khan 1995. Variability in pearl millet. Madras Agric J 82(12): 665-666.

Sharma K.C., Sharma R.K., Singhania D.L. and Singh D. 2003. Variation and character association for fodder yield and related traits in pearl millet (Pennisetum glaucum) Indian J Genet 63(2):115-118.

Singh A., Singh S.K., Sirohi A. and Yadav R. 2009. Genetic variability and correlation studies in green gram Prog agri An Internat J 9(1): 59-62.

Singh B., Upadhyay P.K. and Sharma K.C. 2014. Genetic variability, correlation and path analysis in pearl millet (Pennisetum glaucum L.) Indian Res J Genet \& Biotech 6(3): 491-500.

Singh J., Ranwah B.R., Chaudhary L., Lal C., Dagla M.C. and Kumar V. 2013. Evaluation for genetic variability, correlation and path coefficient in mutant population of forage sorghum (Sorghum bicolar moench) The Bioscan 8(4): 14711476.

Suthamathi P. and Dorairaj M.S. 1995. Variability, heritability and genetic advance in fodder pearl millet Madras Agric J 82(4): 240-243.

Vetriventhan M. and Nirmalakumari A. 2007. Studies on variability parameters in pearl millet (Pennisetum glaucum (L.) R. Br.) Madras Agric J 94:118-120.

Vidyadhar B., Chand P., Devi I. S., Reddy M.V.S. and Ramachandraiah D. 2007. Genetic variability and character association in pearl millet [Pennisetum glaucum (L.) R. Br.] and their implications in selection Indian $\mathbf{J}$ Agric Res 41(2):150-153.

Yadav G.C., Singh P.K. and Singh B.B. and Verma R. 2001. Genetic variability and path coefficient in urd bean Indian J Pulses Res 14: 143-144.

\section{How to cite this article:}

Om Vir Singh, R. Gowthami, Kartar Singh and Neelam Shekhawat. 2018. Assessment of InterCharacters Associations in the Germplasm of Pearl Millet [Pennisetum glaucum (L.) R. Br.] Over Five Years in Hot Arid Climate of Rajasthan, India. Int.J.Curr.Microbiol.App.Sci. 7(01): 3133-3149. doi: https://doi.org/10.20546/ijcmas.2018.701.372 